\title{
Existence and localization of surface states on Fibonacci quasicrystals: A tight-binding study
}

\author{
E. S. Zijlstra, A. Fasolino, and T. Janssen \\ Institute for Theoretical Physics, University of Nijmegen, Toernooiveld, NL-6525 ED Nijmegen, The Netherlands
}

(Received 19 June 1998)

\begin{abstract}
Surface states of the Fibonacci chain are studied within the tight binding off-site model for clusters and approximants by numerical and transfer matrix methods. In each gap of the bulk spectrum a surface state can exist. The existence and energy of a surface state depend on the boundary conditions at the surface. The exponential envelope of surface states is used to define a localization length, which is shown to depend only on the energy of a specific state. [S0163-1829(99)05901-9]
\end{abstract}

\section{INTRODUCTION}

Since the discovery of quasicrystals (QC's) in $1984,{ }^{1}$ it has been an important theme to find out which special electronic properties their structure, which is intermediate between that of crystals and amorphous materials, implies. Many experimental and theoretical results have been obtained for the bulk, ${ }^{2,3}$ but knowledge about QC surfaces is so far limited. ${ }^{4,5}$ About the structure there is information from, e.g., low-energy electron diffraction (LEED) (Refs. 6,7) and scanning tunneling microscopy (STM) (Refs. 8-10) experiments. A theoretical approach towards electronic properties of QC surfaces is, to our knowledge, still missing. In this paper we make a step towards a theoretical understanding of QC surfaces by analyzing surface states of a semi-infinite Fibonacci chain (FC), which is the one-dimensional prototype of a QC.

Surface states in crystals and QC's have an exponential envelope in the bulk. Therefore crystals and QC's can in principle be treated on the same footing, as will be argued in the following. It is well known that in a gap of the electronic spectrum of a lattice periodic crystal a surface state can appear, and that it can be characterized by a complex value of the crystal momentum, which is a function of energy only, and does not depend on the boundary conditions at the surface. All gap states compatible with the bulk structure, independent of the boundary conditions, also called virtual induced gap states, can in this way be characterized by the dispersion $k(E)$, with $k$ complex. Which of these virtual induced gap states then actually exist depends on the boundary conditions at the surface. Due to the lack of periodicity, the crystal momentum is not well defined in a QC. Fortunately, for a surface state in a crystal the imaginary part of the complex crystal momentum is, for a given lattice parameter, inversely proportional to the decay length $l$ of the exponential envelope of the wave function, which is well defined in both crystals and QC's. Therefore the localization length relation $l(E)$ can be extended to QC. It is the goal of this paper to obtain this relation for a model QC.

This paper is organized as follows. In Sec. II the tight binding model Hamiltonian and approximants of the FC are given. Section III is a brief summary of the main previously reported properties of bulk and surface states in the FC. In Secs. IV and V the method of transfer matrices and a numerical diagonalization method for semi-infinite periodic chains (approximants) are treated. In Sec. VI we give the dispersion $l(E)$ and also some other results. We conclude in Sec. VII.

\section{MODEL}

The FC can be formed by substitution rules, starting from a seed. If the substitution rules are applied a finite number of times, the resulting structure is called the unit cell of an approximant of the FC. The number of iterations gives the generation. The actual quasiperiodic FC corresponds to generation $\infty$. The first generation approximant, or seed, $w_{1}$ is given by the word $L$. The substitution rules are $L \rightarrow L S, S$ $\rightarrow L$. The first unit cells of approximants are $w_{1}=L, w_{2}$ $=L S, w_{3}=L S L, w_{4}=L S L L S$. The lengths of these unit cells are given by the Fibonacci numbers, $\left|w_{i}\right|=F_{i}, F_{0}$ $=1, F_{1}=1, F_{j+1}=F_{j}+F_{j-1}$. For the study of electronic properties a nearest neighbor tight binding Hamiltonian is considered:

$$
H=\sum_{i}|i\rangle V_{i}\langle i|+| i\rangle T_{i}\langle i+1|+| i+1\rangle T_{i}\langle i|,
$$

where $|i\rangle$ is a Wannier state localized at the $i$ th site. There are two generally studied models, which differ in the choice of parameters. In the off-site model the on-site energy $V_{i}$ is constant and the hopping terms $T_{i}$ take the values $T_{L}$ and $T_{S}$, which are arranged according to (an approximant of) the FC. In the on-site model the hopping terms are constant and the on-site energies take values $V_{L}$ and $V_{S}$, arranged according to the sequence of the FC. The two kinds of models show qualitatively the same kind of properties, independent of the choice of parameters, ${ }^{11,12}$ except for trivial choices, e.g., $T_{L}$ $=T_{S}, V_{i}=0$, which are the parameters of a crystal. We focus on the off-site model with fixed bulk parameters (hopping terms). The given results were checked numerically to hold as well for different parameter values in the off-site model.

In this paper surface states of a truncated FC are studied by means of (i) transfer matrices, (ii) direct diagonalization of clusters, and (iii) a numerical method for approximants. The studied model is the off-site model with $T_{L}=1, T_{S}=2$, general on-site energy at the surface atom $V_{1}=\alpha$, and zero on-site energy throughout the bulk $V_{i}=0, i \neq 1$. The parameter $\alpha$ is introduced to account for different boundary condi- 


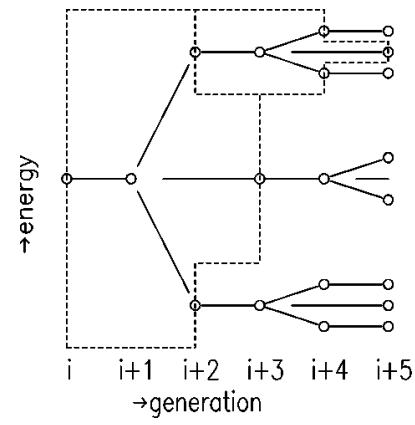

FIG. 1. Band splitting pattern as seen in approximants (Ref. 12). A circle corresponds to an electronic bulk band. The vertical axis gives the energy. On the horizontal axis there are approximants of increasing generation. It is seen that out of one band two side bands are formed after two generations. One central band is formed after three generations. Then the splitting pattern repeats. The gap in the $(i+2)$ th generation approximant is transient. In the $(i+3)$ th generation approximant two stable gaps open.

tions at the surface. As the FC has no translational symmetry, it must of course always be specified where the surface is. Note that this model is the Tamm model for surface states if the first generation approximant is studied. It is the Shockley model if the second generation is studied. These models were the first attempts to understand crystal surfaces. ${ }^{13}$

\section{BULK PROPERTIES}

Numerical $^{14}$ and analytical ${ }^{11,12,15-19}$ studies have revealed much about the electronic bulk spectrum of the FC. Numerical calculations are either performed on a large but finite piece of the FC with free or fixed boundary conditions, the so called cluster model, or on an infinite periodic approximant, the approximant model. This latter model corresponds to an infinite chain that is periodic, with one of the unit cells introduced in Sec. II. In both cases the spectrum and the wave functions may be obtained by direct diagonalization of a Hermitian matrix (Hamiltonian). Analytical calculations were done within the method of transfer matrices ${ }^{11,15-18}$ and with a renormalization group technique. ${ }^{12,19}$ Both methods exploit the inflational symmetry of the FC. In Sec. IV the method of transfer matrices is reviewed. It is then extended for finding the dispersion of virtual induced gap states of the FC, $l(E)$.

The spectrum of the infinite FC within the off-site model is a Cantor set. ${ }^{11,20}$ In the off-site model with the parameters as given in Sec. II the spectrum consists of three main bands, each of which has a substructure of three bands, etc. ${ }^{11,12}$ So there is an infinite number of bands, which are arranged hierarchically in the spectrum. Approximants have a finite number of bands. When going from one approximant to a higher one, the band splitting is taking place in the way of Fig. 1. All wave functions in the bulk of the infinite FC are critical.

In $1990 \mathrm{Niu}$ and $\mathrm{Nori}^{12}$ found that if in the off-site model at one site in an infinite FC the on-site energy is changed by an amount $\Delta V$, i.e., if an impurity is introduced at that site, a state localized near the impurity, and exponentially damped into the bulk, appears in each gap. For $\Delta V \rightarrow \infty$ the state in a given gap will be localized either to the left or to the right of

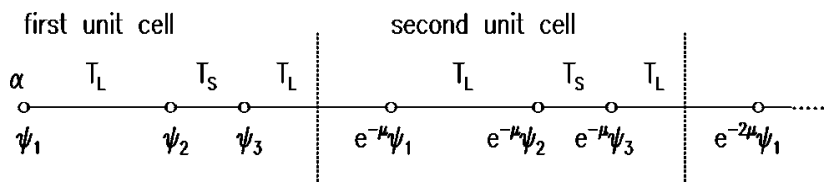

FIG. 2. Surface of the third generation approximant of the Fibonacci chain, starting with $w_{3}$. The surface is to the left. The length of one unit cell $\left|w_{3}\right|$ is $\left|w_{3}\right|=F_{3}=3$. For an eigenstate of the transfer matrix $M_{3}$ at an energy in a gap of the spectrum the wave function is exponentially decaying into the bulk $\psi_{N+n}= \pm e^{-\mu} \psi_{n}$, Eq. (7). In the figure the positive sign was chosen for convenience.

the impurity, except when symmetry requires equal probabilities at both sides. In the limit $\Delta V=\infty$ there are two semi-infinite chains which are not connected. In a given gap of the energy spectrum one of these chains has a localized state. So, in general, if the FC is truncated, i.e., if a zerodimensional surface is created, in about half of the gaps in the energy spectrum a state localized near the surface, with an exponential envelope, appears. These states are called surface states. ${ }^{12,14,19,21,22}$ As a natural length scale for a surface state in a given gap Niu and Nori ${ }^{12}$ proposed the length of the unit cell of the smallest approximant in which this gap appears in the spectrum. This natural length scale will be shown to be quite useful for the general case, when the parameters of the model are not fixed. When the parameters are known it will be shown that a better estimate can be made.

In 1995 Piéchon et al. $^{23}$ pointed out that there are two kinds of gaps in the spectrum of an approximant. A so-called transient gap is found within a band that has just split into two bands, but not yet into three. Such a gap closes in the next generation approximant, when the corresponding central band appears in the spectrum. Stable gaps are gaps that persist for higher generation approximants. This is shown in Fig. 1. In the method of approximants it will be seen that surface states that appear in stable gaps are usually found also in higher generation approximants, whereas states in transient gaps disappear in all higher generation approximants.

\section{TRANSFER MATRICES}

The off-site model can be described by transfer matrices ${ }^{11,17} M_{j}$, which are determined recursively,

$$
M_{1}=\left(\begin{array}{cc}
\frac{E}{T_{L}} & -1 \\
1 & 0
\end{array}\right), \quad M_{2}=\left(\begin{array}{cc}
\frac{E^{2}}{T_{L} T_{S}}-\frac{T_{S}}{T_{L}} & -\frac{E}{T_{S}} \\
\frac{E}{T_{S}} & -\frac{T_{L}}{T_{S}}
\end{array}\right),
$$

$M_{j+1}=M_{j-1} M_{j}$. Let $|\psi\rangle=\sum_{i=1}^{\infty}|i\rangle \psi_{i}$ be an eigenstate of the Hamiltonian (1). As shown in Fig. 2 the sites are labeled starting from the surface, so that $|1\rangle$ is the Wannier state at the surface atom. If $\psi_{1}$ and $\psi_{2}$ are given, the wave function at a Fibonacci number site follows from

$$
\left(\begin{array}{c}
\psi_{F_{j}+2} \\
\psi_{F_{j}+1}
\end{array}\right)=M_{j}\left(\begin{array}{l}
\psi_{2} \\
\psi_{1}
\end{array}\right), \quad j \neq 1
$$


Note that the transfer matrices still depend on the energy $E$. Each transfer matrix $M_{j}$ has unit determinant and real trace. Therefore the eigenvalues of $M_{j}$ for a given energy $E$ are $e^{i k}$ and $e^{-i k}$ with $k$ real or $\pm e^{i k}$ and $\pm e^{-i k}$ with $k$ imaginary. ${ }^{24}$ $k$ real corresponds to bulk states of the given approximant, where $k$ depends on the energy, $k=k(E)$, which is the dispersion relation for a bulk band. Let the bulk solutions for a certain energy be given by $|k\rangle$ and $|-k\rangle$. When the bulk is truncated, i.e., a surface is formed, there is an extra condition for the wave function at the boundary, the so called boundary condition, which is the condition $\langle 1|H| \psi\rangle=\langle 1|E| \psi\rangle$ or

$$
T_{1} \psi_{2}=(E-\alpha) \psi_{1} .
$$

For bulk states with a given energy there exists always a linear superposition $A|k\rangle+B|-k\rangle$ satisfying Eq. (4). At a band edge Eq. (3) has only one eigenstate ${ }^{24}$ with eigenvalue $e^{i k}, k=0$, or $k=\pi$. The energies that give imaginary $k, k$ $=i \mu$ with $\mu$ real, correspond to gaps in the bulk spectrum.

Let $|\mu\rangle(|-\mu\rangle)$ be the wave function that increases (decreases) exponentially when going from the surface into the bulk. The exponentially increasing wave function $|\mu\rangle$ is unphysical. If there were no surface the wave function $|-\mu\rangle$ would be unphysical as well. If the chain is truncated, the state $|-\mu\rangle$ appears in the spectrum if the boundary condition (4) is satisfied. From Eq. (4) it is clear that for every energy $E$ in the gap this happens for exactly one value of $\alpha$, so that, by probing all values of the parameter $\alpha$, an occupied state will be found at any energy within a gap, see Fig. 3. The dispersion relation of the virtual induced gap states, $\mu$ $=\mu(E)$, can be obtained by exploiting this property, e.g., by numerically solving the Hamilton eigenvalue equation for sufficiently many values of $\alpha$. This dispersion relation can also be found by using a recursion relation for the traces of the transfer matrices. This procedure has been used for bulk states; ${ }^{11}$ here it is extended to surface states. Let $x_{j}$ $=\frac{1}{2} \operatorname{tr} M_{j}$. The first values $x_{1}, x_{2}$, and $x_{3}$ are found by taking the trace of $M_{1}, M_{2}$, and $M_{3}$. Then $x_{j+1}=2 x_{j} x_{j-1}$ $-x_{j-2}$. In the $j$ th approximant $\mu$ is given by $x_{j}=\cosh \mu$. For a given approximant $\mu$ is a well defined quantity, but in the limit of the aperiodic FC, i.e., $j \rightarrow \infty$, it goes to infinity. For this reason it is better to work with the localization length $l_{j}$, which for a gap state in the $j$ th approximant is defined as

$$
l_{j}=\frac{F_{j}}{\mu} .
$$

This definition is such that for an exponential wave function $\psi_{x} \propto \exp \left(-x / x_{0}\right)$, it gives the usual decay length $x_{0}$. Numerical calculations show that $l_{j}$ has a limiting value $l_{\infty}$, which is not so surprising since gap states are well known to have an exponential envelope, see Sec I. The relation $l_{j}=l_{j}(E)$ is, by virtue of Eq. (5), for every approximant $j$ equivalent to the dispersion relation of virtual induced gap states, as was already pointed out in Sec I. The relation $l_{\infty}=l_{\infty}(E)$ can now be regarded as the dispersion relation of virtual induced gap states for the aperiodic FC. Note that this dispersion relation does neither depend on $\alpha$ nor on where the surface is, whereas the boundary condition (4) depends on both the surface parameter $\alpha$ and, through $\psi_{1}, \psi_{2}$, and $T_{1}$, on where the surface is.

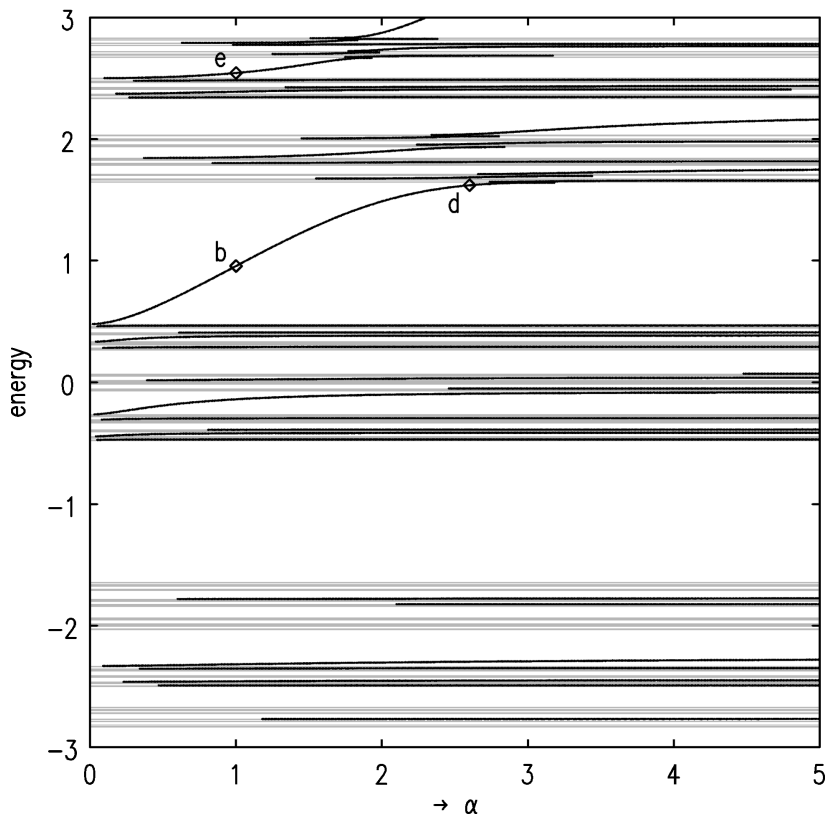

FIG. 3. The spectrum of the ninth generation approximant Fibonacci chain, with 55 atoms per unit cell, with parameters $T_{S}=2$ and $T_{L}=1$, as a function of $\alpha$. The chain starts with $w_{9}$. The grey bands indicate the bulk continuum. Note the hierarchical splitting pattern of these bands. In the gaps surface states are indicated by black lines. The spectrum for negative $\alpha$ can be found by rotating $180^{\circ}$ around the point $(\alpha$,energy $)=(0,0)$. For every energy in a gap there is one $\alpha$ that makes the boundary condition match, as was indicated in the text. For $\alpha=0$ there are no gap states, a proof of which is given in the text. Note further that the energy of a surface state found in a gap is here seen to be a continuously increasing function of $\alpha$, as would be expected from simple perturbative arguments. The letters $b, d$, and $e$ refer to the correspondingly labeled wave functions in Fig. 7.

\section{NUMERICAL METHOD}

Eigenstates $|\psi\rangle=\sum_{i=1}^{N}|i\rangle \psi_{i}$ of a cluster of $N$ atoms are obtained by direct diagonalization of the Hamiltonian. The localization length $l_{c}$ of an eigenstate is defined as the number of sites starting at the surface and going into the bulk that have a summed probability density $1-1 / e$ :

$$
l_{c}=\min \left\{\left.l\left|\sum_{i=1}^{l}\right| \psi_{i}\right|^{2} \geqslant 1-\frac{1}{e}\right\},
$$

where $\psi$ is assumed to be normalized. If the amplitudes $\psi_{i}$ decay exponentially, i.e., $\psi_{x} \propto \exp \left(-x / x_{0}\right)$, then $l_{c}$ is the decay length $x_{0}$.

Bulk states of approximants are obtained by direct diagonalization of the Hamiltonian of one unit cell with periodic boundary conditions. Surface states of approximants can be found by using the property that gap states in a onedimensional nearest neighbor tight binding model are exponentially decaying from the surface into the bulk,

$$
\psi_{n+N}=\left( \pm e^{-\mu}\right) \psi_{n},
$$

where $N$ is the number of sites in one unit cell and $\mu$ is real and is required to be greater than $0, \mu>0$, as pointed out in 
Sec. IV. The objective is to find all surface states for a given surface with given $\alpha$. Two cases can be distinguished.

Case $1, \alpha=0$. The boundary condition at the surface (4), Eq. (7), and the equation $\langle 1+N|H| \psi\rangle=\langle 1+N|E| \psi\rangle$ imply $\psi_{N}=0$. Therefore the equations $\langle i|H| \psi\rangle=\langle i|E| \psi\rangle$, $i=1, \ldots, N-1$ decouple from all other equations, $i$ $=N, \ldots$. So finding surface states corresponds to diagonalizing an $(N-1) \times(N-1)$ matrix, which can be done numerically. $\mu$ is given by $\langle N|H| \psi\rangle=\langle N|E| \psi\rangle$ or

$$
\pm e^{-\mu}=-\frac{\psi_{N-1}}{\psi_{1}} \frac{T_{N-1}}{T_{N}}
$$

Case 2, $\alpha \neq 0$. The boundary condition at the surface now implies

$$
\left( \pm e^{-\mu}\right) \alpha \psi_{1}=T_{N} \psi_{N}
$$

This gives $T_{N} \psi_{N+1}=T_{N}\left( \pm e^{-\mu}\right) \psi_{1}=\left(T_{N}^{2} / \alpha\right) \psi_{N}$. This relation can be used to write down an effective Hamiltonian $H^{\text {eff }}$ for the $\psi_{i}$ in the first unit cell:

$$
H_{i j}^{\mathrm{eff}}=H_{i j}+\delta_{i N} \frac{T_{N}^{2}}{\alpha} \delta_{N j},
$$

where $H_{i j}=\langle i|H| j\rangle$. Finding surface states is equivalent to diagonalizing $H^{\text {eff }}$. $\mu$ can be found by substituting the eigenvectors of $H^{\text {eff }}$ in Eq. (9). In both cases the localization length is given by Eq. (5).

\section{RESULTS}

In Fig. 3 the energies of surface states as a function of $\alpha$ for the ninth generation approximant FC are plotted. The surface is to the left of the chain and starts with $w_{9}$. In this figure $\alpha=0$ is a special case, since it gives no surface states at all. This can be understood from symmetry by noting that for the $j$ th generation approximant, with the surface to the left, and starting with $w_{j}$, only the hopping terms corresponding to the reduced word

$$
\tilde{w}_{j}=\left\{\begin{array}{lll}
w_{j} S^{-1} L^{-1}, & j & \text { even, } \\
w_{j} L^{-1} S^{-1}, & j & \text { odd },
\end{array}\right.
$$

are important for the wave function $\psi_{1} \cdots \psi_{N}$ in the first unit cell, as indicated in Sec. V, Case 1. Here $S^{-1} L^{-1}$ or $L^{-1} S^{-1}$ denotes the removal of two letters at the end of the word $w_{j}$. The damping factor $\left( \pm e^{-\mu}\right)$ is given by Eq. (8),

$$
\left( \pm e^{-\mu}\right)= \begin{cases}-\frac{\psi_{N-1}}{2 \psi_{1}}, & j \text { even, } \\ -\frac{2 \psi_{N-1}}{\psi_{1}}, & j \text { odd }\end{cases}
$$

It will be shown that $\tilde{w}_{j}$ is a symmetric word. Therefore $\psi_{N-1} / \psi_{1}= \pm 1$ for all surface states. For $j$ even there are thus $N-1$ surface states, one in each gap. The localization length of all states is equal. It is proportional to the size of the unit cell $l_{j}=F_{j} / \ln 2$. So the localization at the surface is less and less for increasing generation $j$. For $j$ odd there are no surface states, since $e^{-\mu}=2>1$. From this it is clear that the approximant $w_{\infty}$ starting with $w_{\infty}$, and with $\alpha=0$, has no localized surface states. The proof that $\tilde{w}_{j}$ is symmetric goes by induction. First of all it is easy to check that $\tilde{w}_{3}, \tilde{w}_{4}$, and $\tilde{w}_{5}$ are symmetric. Then because $\tilde{w}_{j}=\tilde{w}_{j-2} L S \tilde{w}_{j-3} S L \tilde{w}_{j-2}$ for $j$ even, and $\tilde{w}_{j}$ $=\tilde{w}_{j-2} S L \tilde{w}_{j-3} L S \tilde{w}_{j-2}$ for $j$ odd, $\tilde{w}_{j}$ is obviously symmetric for all $j$.

In order to make sure that the absence of surface states at this specific surface for $\alpha=0$ is not an artifact of the method of approximants the same surface was also studied with the cluster method. Clusters which consist of the first $N$ sites of the FC, starting with $w_{\infty}$, were taken, with $N$ $=1, \ldots, 2500$. The localization length $l_{c}$, of the best localized state in each of these clusters is plotted in Fig. 4 as a function of the cluster size $N$. It is seen to increase approximately linearly with cluster size, which makes it probable that there are no surface states at all.

For general $\alpha$ about half of the gaps have a surface state. At $\alpha= \pm \infty$ there is an eigenstate fully localized on the first site. It doesn't interact with the rest of the chain, which then starts with $L^{-1} w_{9}$, with $\alpha=0$, where $L^{-1}$ indicates the removal of one atom from the surface of the chain. This conclusion was checked numerically. Other surfaces give similar results. When the chain starts at a generic point in the unit cell of an approximant, there is no value of $\alpha$ where all surface states disappear. It is important to notice that the number of surface states depends on $\alpha$.

Figures 5 and 6 show the localization length $l_{16}$, Eq. (5), as a function of the energy. In general it is hard to predict what boundary conditions a physical system has at the surface. Figure 5 indicates what is possible for various bound-

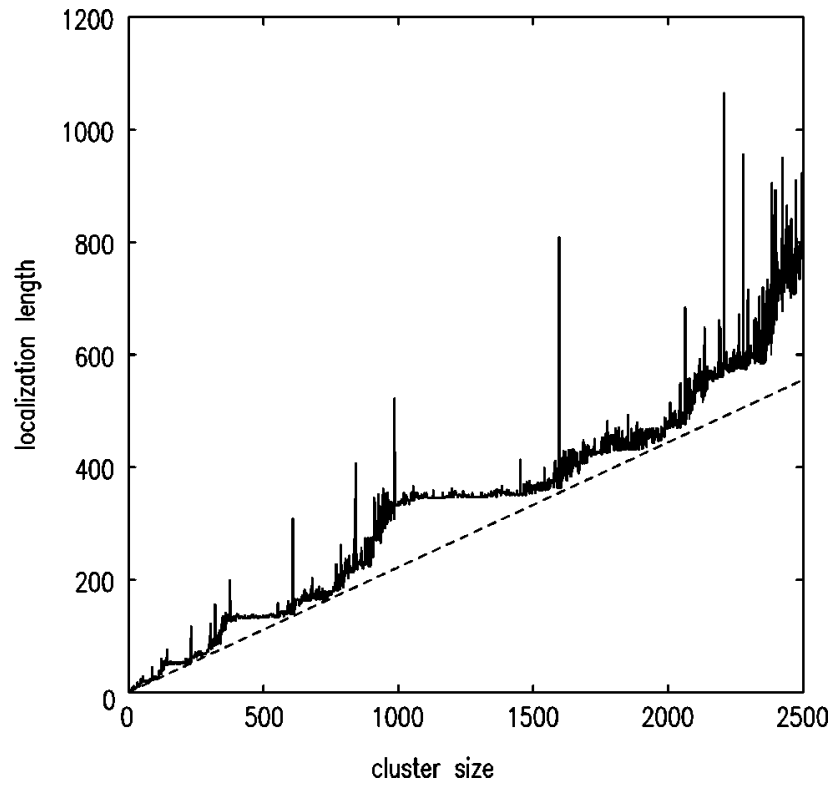

FIG. 4. The localization length $l_{c}$ of the best localized state for the left surface of the clusters $L, L S, \ldots, w_{\infty}$ as a function of the cluster size $N$, with $\alpha=0$. Note the self-similarity in the graph, which can only appear because there are no surface states. The highest peaks correspond to symmetric words, which have symmetric and antisymmetric states only. The dashed line is the function $l_{c}=N / 4.5$. It is a lower estimate for the localization length. As this function is linear in the cluster size, it is probable that the left surface of $w_{\infty}$ has no surface states at all. 


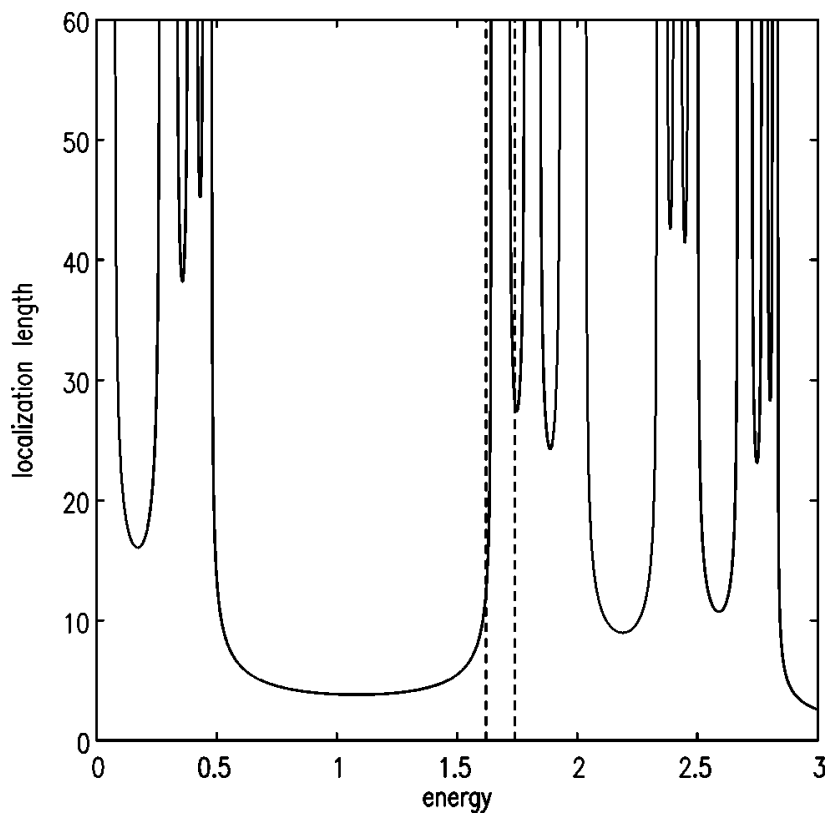

FIG. 5. The localization length $l_{16}$ as a function of energy in the 1597 atoms per unit cell approximant with $T_{L}=1$ and $T_{S}=2$. All shown points have converged to the infinite Fibonacci chain value. In a large part of each gap $l_{j}$ is close to its minimum value in the gap. Therefore it is useful to define as a typical localization length for a surface state in a gap its minimal possible value in this gap. This typical localization length then depends on the ratio $\left|T_{L} / T_{S}\right|$. The energy range between the dashed lines is shown enlarged in Fig. 6.

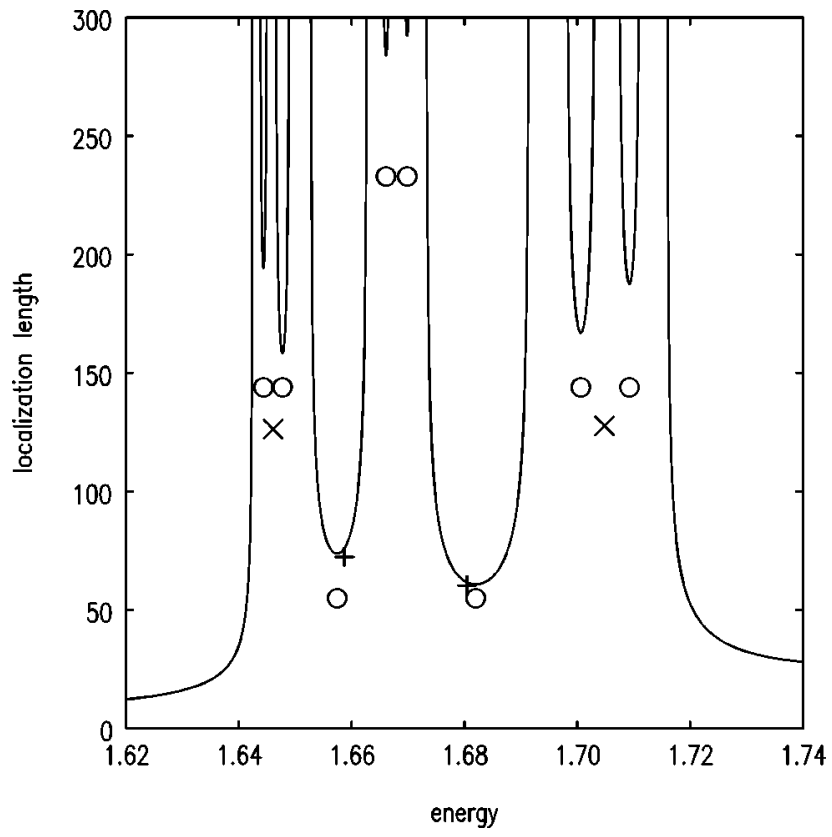

FIG. 6. The localization length $l_{16}$ as a function of energy in the 1597 atoms per unit cell approximant with $T_{L}=1$ and $T_{S}=2$ in a small energy range. All shown points have converged to the infinite Fibonacci chain value. The typical length scales as proposed by Niu and Nori (Ref. 12) for the gaps in this picture are indicated by pluses. For the ratio $\left|T_{L} / T_{S}\right|=0.5$ it is quite good as a rough estimate. The minimal localization length $l_{10}$ of the 89 atoms per unit cell approximant is given by pluses (stable gaps) and crosses (transient gaps). The states present in transient gaps are not present in the 16th generation approximant.

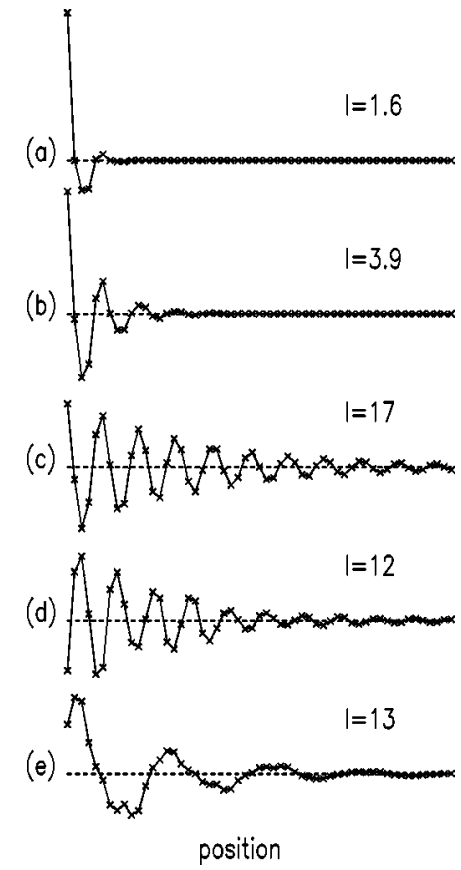

FIG. 7. Surface states of the ninth approximant of the Fibonacci chain, starting with $w_{9}$. The localization length $l_{9}$ is given for each of these states. (a)-(c) is the surface state in the biggest gap of the spectrum for $\alpha=1$, and different values of the bulk parameters: $T_{S} / T_{L}=5,2$, and 1.2 , respectively. It is seen that the localization is stronger for large $T_{S} / T_{L}$, as is indicated in the text. (d) is the state that appears in the biggest gap when $\alpha=2.6$, and $T_{S} / T_{L}=2$. In the energy spectrum it lies closer to a bulk band than state (b), see Fig. 3 , and it has therefore a longer localization length, as follows from Figs. 4 and 5. (e) appears for the same parameters as state (b), but in another gap, which is indicated in Fig. 3. It also has a longer localization length than state (b).

ary conditions as far as the bulk is concerned. The minimum localization length in the biggest gap in the spectrum, e.g., is approximately 3 atoms. In Fig. 6 the length scale of Niu and Nori $^{12}$ is seen to hold quite well for the specific ratio $\left|T_{L} / T_{S}\right|=0.5$, as a rough estimate. This indicates that the conclusion of Niu and Nori, ${ }^{12}$ that surface states will roughly be found with a hierarchy of localization lengths, is true. However the exact length scales depend on the ratio $\left|T_{L} / T_{S}\right|$, and on the boundary conditions. In the caption of Fig. 6 the minimum localization length within a gap is proposed as a better typical length scale. This definition depends on $\left|T_{L} / T_{S}\right|$. In fact in the limit of a periodic crystal, $\left|T_{L} / T_{S}\right| \rightarrow 1$, this better typical length scale goes to infinity, because in the limit all states will be extended. In the other limit, $\left|T_{L} / T_{S}\right| \rightarrow 0$, the chain splits into atoms and bi-atoms, and all states are infinitely well localized. Typical length scales should go to zero. These ideas are illustrated by Fig. 7 , where wave functions of the ninth approximant FC are shown for various bulk and surface parameters.

\section{OUTLOOK}

We have shown how the spectrum of virtual induced gap states can be defined and calculated for the FC. Surface states are for moderate ratios $\left|T_{L} / T_{S}\right|$ seen to be approximately localized according to a hierarchy of length scales. In 
the generic case in about half of the gaps a surface state is found. This means that quite many electrons in a QC might be trapped in surface states. However, it is nontrivial to indicate the implications of this result for physical properties. Two extensions of the present work are quite natural. First of all the effect of randomization of the lattice might be important, since there are indications that real QC's have some degree of randomness in their structure. Furthermore, to study real QC's it is necessary to study higher dimensional models, where surfaces have structure, because bonds can be cut according to different prescriptions. This might reflect different ways in which real QC surfaces can be prepared in experiments. The complexity of the results in this simple model shows that studies in higher dimensions should be carried out with care.

\section{ACKNOWLEDGMENT}

The authors thank Michael Baake for fruitful discussions.
${ }^{1}$ D. Shechtman, I. Blech, D. Gratias, and J. W. Cahn, Phys. Rev. Lett. 53, 1951 (1984).

${ }^{2}$ S. Roche, G. Trambly de Laissardière, and D. Mayou, J. Math. Phys. 38, 1794 (1997).

${ }^{3}$ Z. M. Stadnik, D. Purdie, M. Garnier, Y. Baer, A.-P. Tsai, A. Inoue, K. Edagawa, S. Takeuchi, and K. H. J. Buschow, Phys. Rev. B 55, 10938 (1997).

${ }^{4}$ New Horizons in Quasicrystals, Proceedings of the International Conference, edited by A. I. Goldman, D. J. Sordelet, P. A. Thiel, and J.-M. Dubois (World Scientific, Singapore, 1997).

${ }^{5}$ Jean-Marie Dubois, in Introduction to Quasicrystals, edited by J.-B. Suck et al. (Springer Verlag, Berlin, in press).

${ }^{6}$ M. Gierer, M. A. Van Hove, A. I. Goldman, Z. Shen, S.-L. Chang, C. J. Jenks, C.-M. Zhang, and P. A. Thiel, Phys. Rev. Lett. 78, 467 (1997).

${ }^{7}$ Z. Shen, C. J. Jenks, J. Anderegg, D. W. Delaney, T. A. Lograsso, P. A. Thiel, and A. I. Goldman, Phys. Rev. Lett. 78, 1050 (1997).

${ }^{8}$ T. M. Schaub, D. E. Bürgler, H.-J. Güntherodt, and J. B. Suck, Phys. Rev. Lett. 73, 1255 (1994).

${ }^{9} \mathrm{Ph}$. Ebert, M. Feuerbacher, N. Tamura, M. Wollgarten, and K. Urban, Phys. Rev. Lett. 77, 3827 (1996).

${ }^{10} \mathrm{Ph}$. Ebert, F. Yue, and K. Urban, Phys. Rev. B 57, 2821 (1998).
${ }^{11}$ Mahito Kohmoto, Bill Sutherland, and Chao Tang, Phys. Rev. B 35, 1020 (1987).

${ }^{12}$ Qian Niu and Franco Nori, Phys. Rev. B 42, 10329 (1990).

${ }^{13}$ E. T. Goodwin, Proc. Cambridge Philos. Soc. 35, 221 (1939); 35, 232 (1939); Sydney G. Davison and Maria Stȩślicka, Basic Theory of Surface States (Clarendon, Oxford, 1992).

${ }^{14}$ Franco Nori and J. P. Rodriguez, Phys. Rev. B 34, 2207 (1986).

${ }^{15}$ Mahito Kohmoto, Leo P. Kadanoff, and Chao Tang, Phys. Rev. Lett. 50, 1870 (1983).

${ }^{16}$ Stellan Ostlund, Rahul Pandit, David Rand, Hans Joachim Schnellnhuber, and Eric D. Siggia, Phys. Rev. Lett. 50, 1873 (1983).

${ }^{17}$ Mahito Kohmoto and Jayanth R. Banavar, Phys. Rev. B 34, 563 (1986).

${ }^{18}$ John A. G. Roberts, Physica A 228, 295 (1996).

${ }^{19}$ Qian Niu and Franco Nori, Phys. Rev. Lett. 57, 2057 (1986).

${ }^{20}$ Hong-ru Ma and Chien-Hua Tsai, J. Phys. C 21, 4311 (1988).

${ }^{21}$ K. Bajema and R. Merlin, Phys. Rev. B 36, 4555 (1987).

${ }^{22}$ Youyan Liu and Rolf Riklund, Phys. Rev. B 35, 6034 (1987).

${ }^{23}$ Frédéric Piéchon, Mourad Benakli, and Anuradha Jagannathan, Phys. Rev. Lett. 74, 5248 (1995).

${ }^{24}$ András Sütö, in Beyond Quasicrystals: Les Houches, March 7-18, 1994, edited by F. Axel and D. Gratias (Springer Verlag, Berlin, 1995), Chap. 17. 